\title{
Enhancing test practicality for in-house English proficiency tests: An efficient item-banking system
}

\author{
Akiyo HIRAI \\ University of Tsukuba
}

\begin{abstract}
This paper will consider the issue of test practicality by explaining an item banking system being developed for an in-house proficiency test at University of Tsukuba. We administer English language placement and year-end achievement tests to all first-year university students every year. In lieu of the demand for many test forms and the need for precise assessment of the students each year, all the test forms consist of items calibrated using Item Response Theory (IRT) and some items are repeatedly used on different test forms. Under these circumstances, efficient item management in the form of an item bank has been increasingly in demand. Since there was little information about practical item banking systems, we decided to use Microsoft ${ }^{\circledR}$ Access software to develop a user-friendly item bank as part of the in-house test reform project.
\end{abstract}

\section{Background}

We have established an assessment system ${ }^{1}$ over the past several years, which is used to handle approximately 2400 freshmen who enter the university each year. As illustrated in Figure 1, a placement test at the beginning of the year divides the incoming students into groups of $\mathbf{4 0}$ to $\mathbf{4 5}$ in each department. Also, the placement test, which consists of newly created items and some anchor items, has the function of pre-testing new test items. Based on the anchor items, the rest of the items are calibrated on the same scale as items stored in the item bank using a Rasch-Model Computer Program called Bigsteps ${ }^{2}$ (i.e., a one-parameter IRT; Linacre \& Wright, 1997).

The one-parameter Rasch model is easy to understand and use, and estimates person ability and item difficulty by analyzing the interaction between test taker and test item simultaneously in an orderly and uniform way. Also, the functioning of this model is robust. As opposed to two- and three-parameter models, the Rasch model assumes that items are equally discriminating, which is unlikely to be the case in most tests. However, in fact it can handle data from items which do not conform to this assumption by detecting such as 'misfit' items in the output from the analysis (McNamara, 1996). 
The end-of-year tests, on the other hand, assess achievement over the academic year. This achievement test is particularly important for students because students below a cutoff point on the test must retake it in subsequent years until they are successful. To provide all students with a chance of passing the achievement tests, additional sittings are provided each year. This naturally increases the burden of creating multiple test forms. In addition to assigning students a pass or fail evaluation, their achievement over the year can be assessed by obtaining the gain scores based on the placement and achievement tests. This is possible as items of both tests are calibrated on the same latent ability continuum. Also, the English level of incoming students in each department over time can be assessed by comparing mean scores for placement tests across different years (Hirai \& Shillaw, 2002).

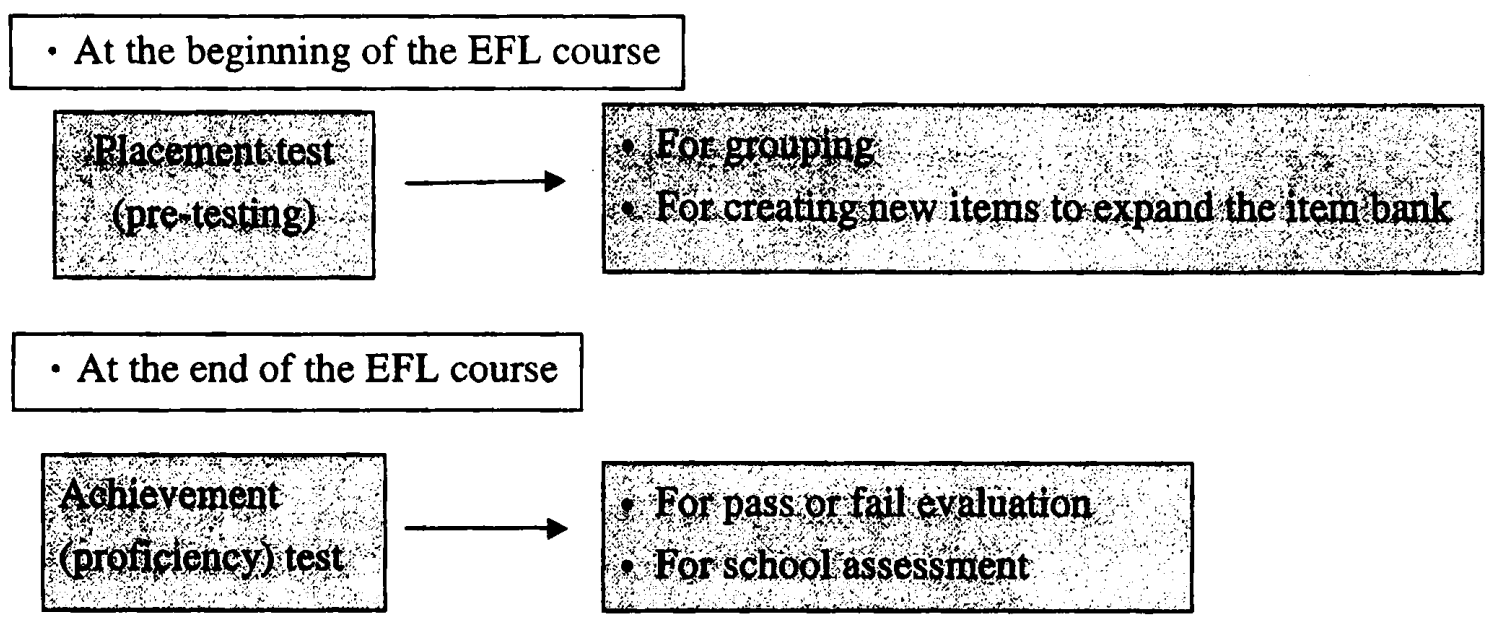

Figure 1. The purpose of placement and achievement tests.

\section{The Purpose of the Present Study}

\subsection{Test Practicality}

The burden of implementing multiple test forms every year was very heavy. Consequently, we have considered increasing test practicality more seriously.

Test practicality is defined as "the extent to which an examination is practicable in terms of the resources required to produce and administer it in its context of use (e.g., The Association of Language Testers in Europe (ALTE) Working Group, 2001, p.15)." It is "an integral part of the concept of test usefulness ${ }^{3}$ and is linked to many different aspects of the examination, including factors such as availability, format and 
sustainability (p.15)." Thus, "a practical examination is one that does not placc unreasonable demands on either the test producer or the test user (p.15)."

In this sense, practicality is all the more important, as test implementation has become part of our routine. Yet, practicality has not often been discussed in the literature of language assessment perhaps because it "pertains primarily to the ways in which the test will be implemented (Bachman \& Palmer, 1996, p.35)," while other test qualities such as validity, reliability, and authenticity are directly related to test scores.

Based on this notion, we looked into the procedure of our testing system to find steps in which we could increase test practicality. One idea we came up with was to create a more efficient item banking system which would suit to our needs.

\subsection{Item Banking}

An item bank is a collection of test items which have been classified and stored in a database so that they can be selected for use in new tests. The items are categorized according to certain descriptive characteristics such as subject area (e.g., listening, reading, or grammar), item type (e.g., multiple-choice, true-or-false, or short-answer items), as well as various pertinent item characteristics (e.g., the item difficulty and item discrimination parameters) (Beeston, 2000; Rudner, 1998).

Usually, all the item difficulties in the item bank have been placed on the same latent ability continuum per subject matter by applying an IRT method. Henning (1986) raises the advantages of using such an item bank for producing multiple forms because "the item bank permits a nearly infinite variety of combinations and permutations of items to be drawn for any specific measurement purpose. This effectively minimizes security problems, since even those examinees with prior exposure to certain items in the bank cannot know what future combinations will appear (p. 73)." Another advantage is that a test form can be efficiently adjusted to the expected test takers' ability levels since the difficulty of each item is known beforehand. This avoids giving redundant items with the same item difficulty or useless items that are too easy or difficult.

The development of item banks has, therefore, been accelerated in accordance with recent advances in computer technology, the spread of e-learning in schools, and computer-based testing such as a web-based test and a computer-adaptive test. Consequently, item bank systems have been used for large scale commercialized tests such as Cambridge.ESOL Exams by UCLES, TOEFL and TOEIC tests by ETS, and CASEC tests by STEP. Smaller private companies and universities (e.g., E-Learning Service; WebLAS system) have been developing e-learning systems (i.e., which have a function of item banking) using open database software such as $M y S Q L$, and PostgreSQL and free educational software such as Moodle. Also, ready-made item 
banking software is available such as FastTEST and MicroCAT by Assessment Systems Corporation Inc. and $L X R * T E S T$ by Applied Measurement Professionals Inc.

However, some software is still expensive to purchase or service; other software is suitable for computer-adaptive tests, but not for paper-and-pencil tests. From the view point of test security and practicality, we are not considering web-based testing at the moment. It is still costly and difficult to administer a high-stakes test to 2400 students in one day. One main reason for not using those item banks is that, at the time our project started several years ago, there was little information about easy-to-use commercialized item banks for paper-and-pencil multiple-choice (MC) format tests or one which allowed us to establish an efficient item banking system for our university at a minimal investment.

Thus, we chose to employ a widely used database program, Microsoft ${ }^{\circledR}$ Access to develop an item bank for our test. There are several reasons why we decided to use this software to create an item bank. First, Access databases can easily import and export data to and from other popular software such as Microsoft ${ }^{\circledR}$ Excel and Word. Second, Access has great potential to create a powerful and convenient database, which can be modified easily as our needs change. Third, under an intra-net system, the same data can be shared among registered members, so that members can work with individual computers. Fourth, it is relatively easy to find technical support on or off campus. In that sense, even if the person in charge becomes unavailable, another person can continue to run and modify the item bank as necessary. Fifth, as opposed to some imported software, Access database allows us to write commands and instructions in Japanese. This makes it easier for Japanese users to use the item bank. Sixth, the item bank may work more efficiently when the new version of this software becomes available. It is expected that things we cannot do or which are cumbersome now will become possible or easier in the future. For all these reasons, Access software was a good option for our testing system.

Thus, the purpose of this study is to develop an efficient item bank using Access database software in order to enhance the practicality of our testing processes. This project started as part of the in-house test reform project in 2002.

\section{Method}

\subsection{Test Format}

Both placement and achievement tests are paper-and-pencil MC tests, consisting of six sections making a total of about 65 items. Short Dialog and Long Dialog/Passage 
sections are for testing listening ability. Vocabulary and Reading Passages mainly measure reading ability. Grammar and Error Detection are for measuring syntactic knowledge. The testing time for all the sections of a test is set for 65 minutes. A test is administered to all freshmen in half a day and test sheets are carefully collected after being administered, so that the same test form may be used again some years later.

\subsection{Reviewing the Process of the Current Testing System}

In the procedure from 1a to $5 \mathrm{a}$ of Figure 2, all the newly created items are pre-tested in a placement test to determine item difficulty of the new items (i.e., calibration). Only good items are stored in the item bank. When a new test form is necessary, approximately 65 items are selected from the item bank and edited into a test form as indicated in the procedure from $1 \mathrm{~b}$ to $5 \mathrm{~b}$.

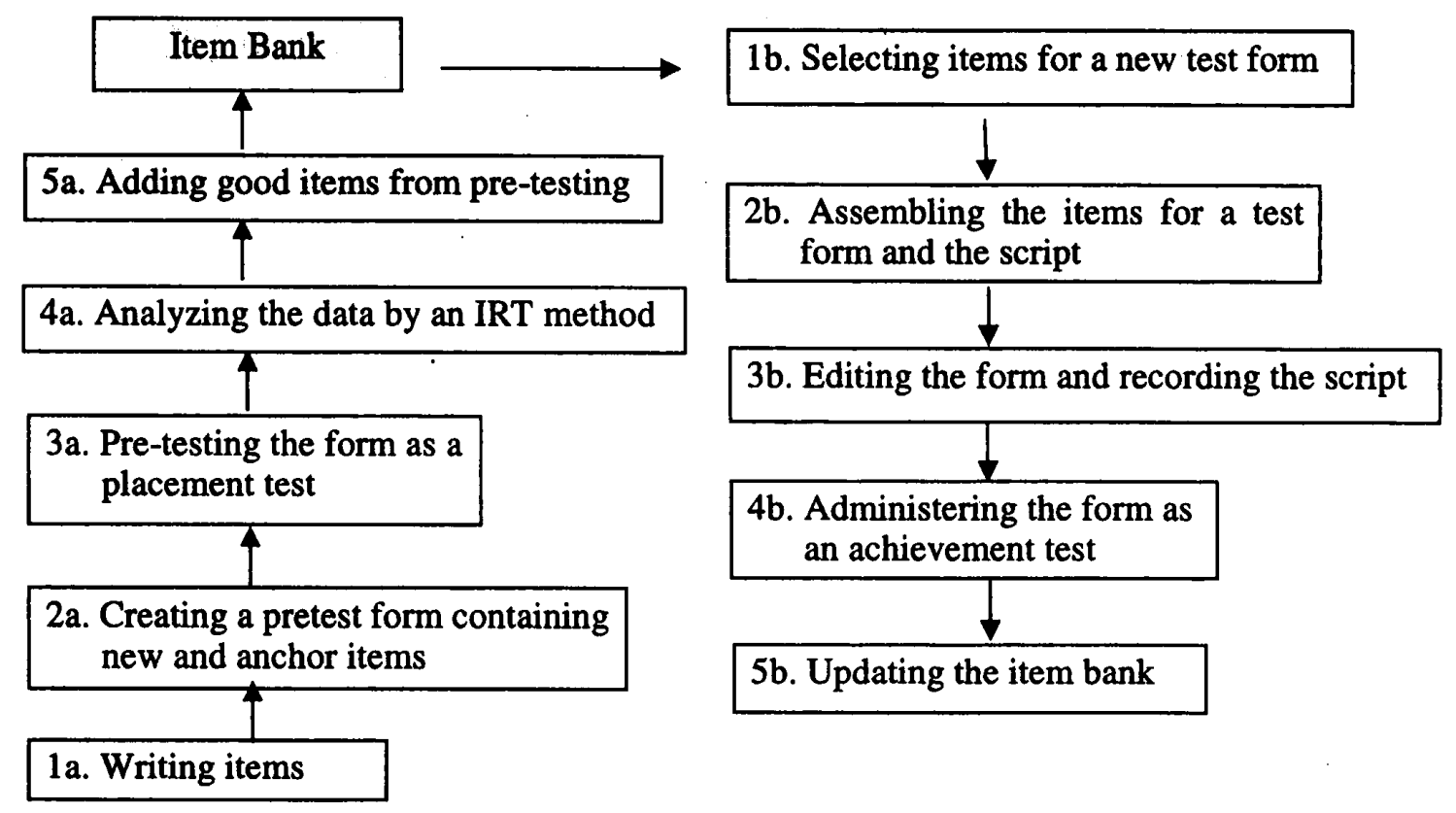

Figure 2. Testing process.

As we have increased the number of items in the bank for multiple test forms, efficient item management such as storing new items in an organized way and recording which ones are being re-used in what test forms has become increasingly important. Also, we wanted to improve the procedure of selecting and editing items for a new test form, since it had taken a great amount of time and energy until a new form was completed.

Keeping these points in mind, we started designing an item bank by learning how 
to use Access software with manuals (e.g., Machida, 2001; Smith, et al., 2003) and by taking an Access basic course for two days. As shown in the colored boxes in Figure 2, the item bank can be used to perform Steps 5a, 1b, 2b, and 5b. Therefore, these steps have been the focus of our attention in developing a user-friendly item banking system.

\section{Developing an efficient item-banking program}

\section{1. (5a) Item Storage}

As briefly explained earlier, each section of the placement test consists of newly created items and a few anchor items. After administering the placement test, all the new items are analyzed by a one-parameter Rasch model. Among them, only items with acceptable fit indices and with effective multiple-choice options (i.e., distractors), are stored into the item bank. Usually, around 40 new items in a placement test are added to the bank.

Previously it had taken much time to store the item data, texts, and scripts in the database as we had to copy and paste them manually. However, using an Access query function, data pertaining to items such as item IDs, item difficulty values, and answers can be automatically imported from an Excel file, in which the necessary results are produced from the IRT analysis, to the item bank. As for storing item texts and listening scripts in the bank each of the texts and scripts is prepared in a separate MS-Word file. Next, with Access macro functions, these texts and scripts are copied into the item bank all at once by simply entering the file names common to all (see Figure 3). In this way, all 40 items of data as well as the texts and the scripts can be stored automatically.

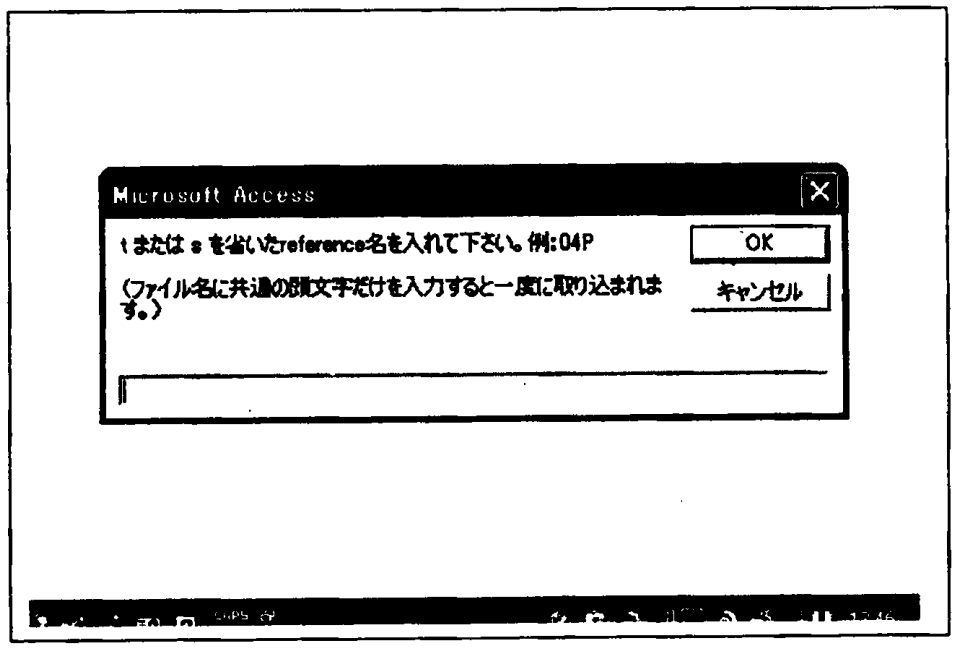

Figure 3. Automatic item texts and scripts storage system. 


\section{2. (1b) Item Selection}

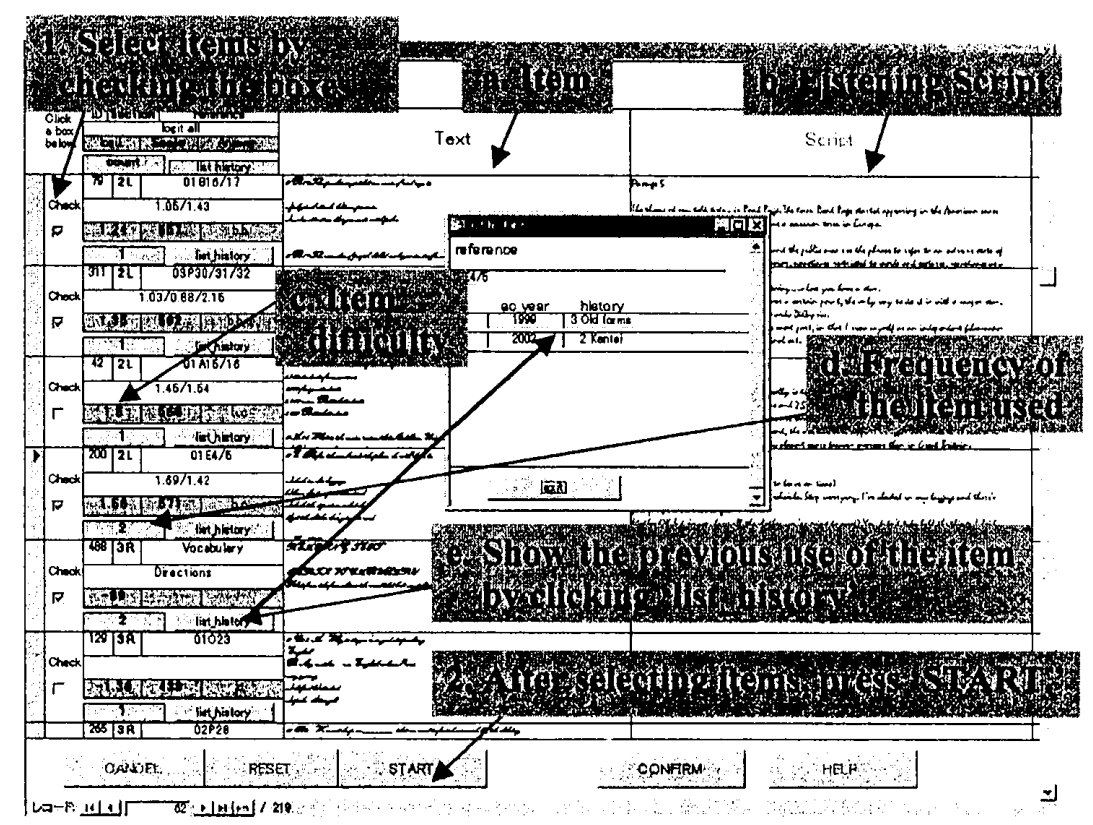

Figure 4. Item selection procedure.

Next, in the item selection stage, a total of about 65 items can be selected easily by simply checking the selection box (see 1 in Figure 4). Items are arranged from Section 1 (Short Listening Dialog) to Section 6 (Error Detection) and, in each section, items are arranged from easy to difficult, which makes the item selection easier. When selecting items from the bank, it is important to carefully consider not only item content (i.e., 'a. Item' and 'b. Listening Script' in Figure 4) but also its level of difficulty 'c.' We have also created the 'frequency' box ' $d$,' which indicates how frequently the item has been used since some items are repeatedly used on different test forms. Moreover, this system makes it possible to easily check when items were used in the past. By clicking on the 'list_history' button 'e,' a small window appears which indicates the year and occasions this item has been used as shown in Figure 4.

\section{3. (2b) Assembling Items for a Test Form}

After selecting the necessary number of items in addition to the test instructions for each section, we click the 'START' button (see 2 in Figure 4). Then, the item bank will automatically create the following three files: (a) a list of the selected items' information (see Figure 5); (b) a test form in a Word document, which consists of the test instructions at the beginning of each section and the items selected, which are 
arranged from easy to difficult based on the item difficulty values; and (c) a list of the listening scripts in another Word document. To view these Word documents, the item bank asks the user to click the 'OK' button which appears in the center of the Figure 5.

Creating a neat test form not requiring editing was a challenge. In an earlier version of the item bank, 65 items were selected from two text boxes: ' $a$. Item' and ' $b$. Listening Script' in the Selection View shown in Figure 4; however, it did not allow us to underline or italicize part of a text in each cell since the text display specification controls all the cells that these texts are in. Therefore, even though these item texts and scripts were selected from cells ' $a$ ' and ' $b$ ' to create a test form, it still required much editing such as referring to the original texts, adding all the italics and underlines manually, and making other format adjustments.

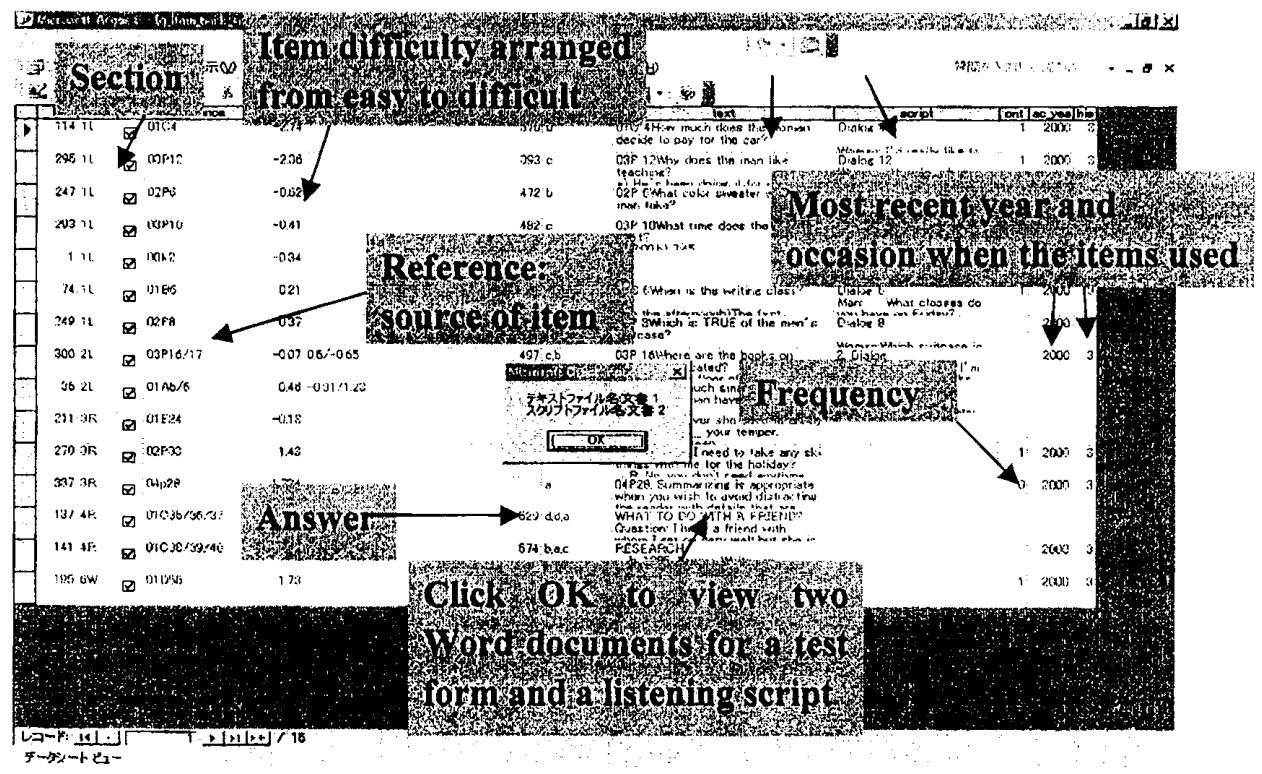

Figure 5. A list of selected items.

This problem was overcome with the help of Access technical support from off campus. We now take the following steps. First, we store each already edited clean text and script with the necessary italics and underlined sections, in each separate Word file as explained in the (5a) Item Storage stage (see Section 4.1). Next, by using the Access hyperlink function, these Word files can link to those corresponding items in the item bank with item identification (which is named 'reference' in this program) through the file pass. Therefore, when we select items by clicking the check boxes and press the 'START' button (see Figure 4), Word files corresponding to the same 'reference' names are opened and the document in each file starts assembling onto a newly created Word 
file. Thus, the final product which consists of these assembled Word documents do not need much editing as they come from the original neat Word documents. In the same way, selected listening scripts are assembled onto another Word file.

For re-selecting items, we only change the check marks or press the RESET button to erase all the checks. The CANCEL button is used to close the window. However, the checks remain when we open the item bank again unless we press the RESET button, which enables us to resume the job from where we last finished.

\section{4. (5b) Updating the Item Bank}

Updating the data in the bank is also an important job for efficient item management. After these 65 items are implemented as a year-end test, we return to the item bank again and press 'CONFIRM.' A small window appears as shown in Figure 6, in which we write the year in 'ac_year' box and select the occasion in 'history' list box in order to keep records of when these items are being used. With the 'OK' button, the data in the 'frequency' and 'list_history' boxes ( $d$ and e in Figure 6) will be updated. In other words, the numbers in the frequency box for all the checked 65 items have increased by one point, for example, from 2 to 3 . Also, the year entered appears in the 'list_history' box for all the checked items when we click the 'list_history' button as indicated in Figure 4. Thus, the next time we create a new test form, we can avoid items that have been frequently, or recently, used.

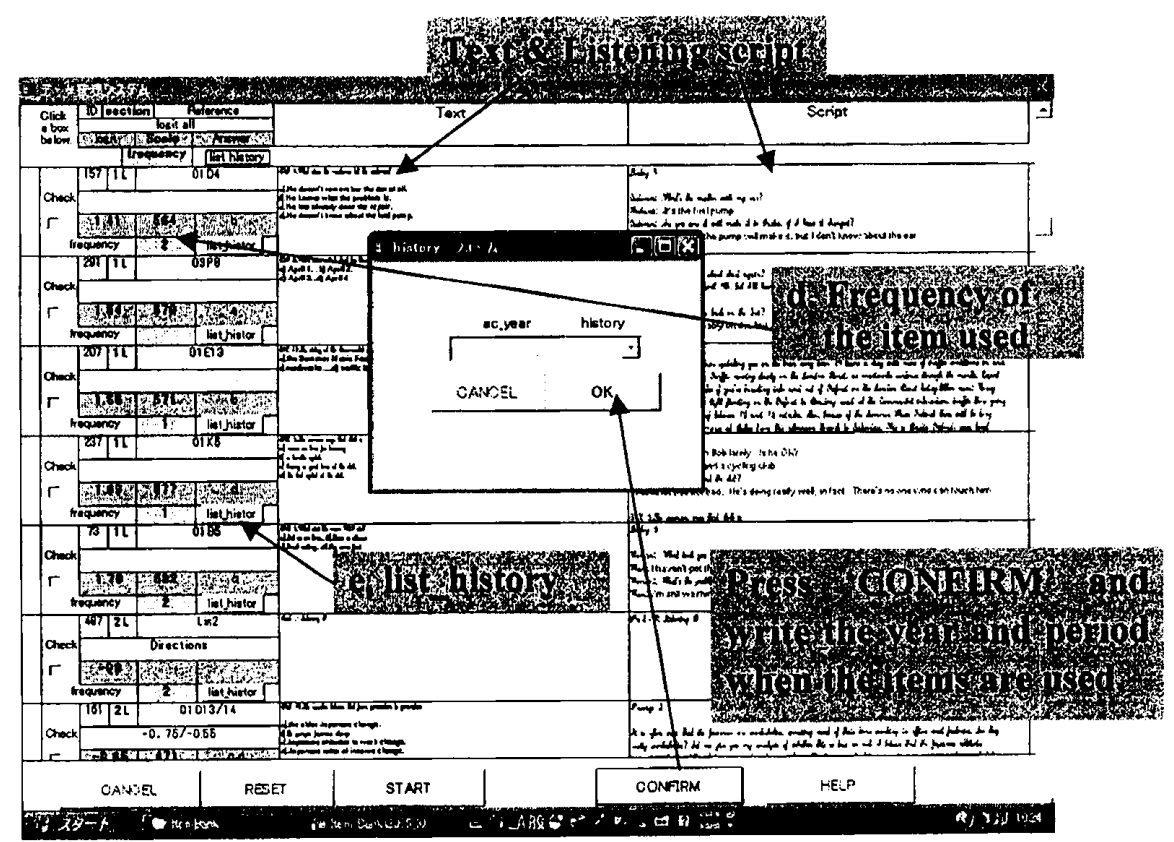

Figure 6. Updating item frequency and item history. 


\section{Results and Conclusions}

The development of a user-friendly item bank has taken three years by trial and error and we have made the following improvements.

First, a number of new item data, texts, and listening scripts can be stored in the item bank quickly without copying and pasting manually.

Second, item selection has been made much easier by just checking the selection box and looking at all the necessary information that appears in the selection view. Then, by clicking a single button, we can obtain two Word documents: one is a test form for test takers and the other is a list of listening scripts for teachers in charge of recording the scripts. In addition to these files, we can access a selected item list with all necessary information, which can be used for item replacement and for scoring as answers of the items are included in the list.

Third, the editing time has been greatly reduced because test items selected for a test form are automatically assembled from neat Word documents. Thus, the test form requires only a minimum amount of editing such as placing a number on each item and adjusting indentations.

Fourth, data updating has become efficient. By simply writing the year and selecting the occasion from the list box for the selected items being used, the items' frequency numbers and history data are updated automatically. Thus, the time spent on data updating has been greatly reduced and we can select items for another test form quickly.

These improvements have enabled us to greatly economize the time and energy spent on the test creation process. In particular, the manipulation of item selection and the creation of test forms have become easier, so that test developers (i.e., teachers) who are not familiar with Access database will be able to handle it easily. This point is important since test creation and implementation is a team effort and jobs are rotated.

Fortunately, we could receive Access technical support when necessary. In this regard, enhancing test practicality is closely related to the advancement of technology and depends on affordable human and financial resources. Therefore, during the planning stage, it is vital to know the following: which technology can be available to help our work, how much we can afford, and who among the members or external people can handle it. 


\section{Future Issues and Implications}

Our item bank still needs some improvements. First of all, we have to enhance management of listening and reading texts with plural questions, which need to be treated differently from other discrete items (i.e., one item with one question such as vocabulary and grammar items). At present, we manually store a text and items attached to it as a group in the bank because these items need to be selected altogether. Secondly, it would be easier to select items if these are further sub-categorized instead of the current six-section categorization. In the grammar section, for example, there are a variety of items such as ones testing the knowledge of prepositions, relative clauses, articles and infinitives. By categorizing grammatical items based on these grammatical functions and arranging them in order, we will avoid selecting items asking similar grammatical points and cover a wider range of grammatical knowledge, which will result in increasing the validity of our test. Third, as the number of items stored in the bank is increasing, it is troublesome scrolling up and down to go to a different section for item selection. Therefore, it would be convenient if we could jump to the beginning of each section immediately.

In order to make the item bank more user-friendly, we will continue working on solving these problems. At the same time, we will be developing an automatic test item assembly program which can be used by other teachers in any subject area who want to keep their own test items for reuse. This would be possible since most of the functions we have developed are the ones that would be prerequisite in establishing any item banking system.

Besides improving the container of the item bank, in the future, when increasing number of items repeatedly appear in a different form, we may need to investigate the stability of the logit value and fit statistics for banked items and the number of exposures it takes to substantially change these parameters for different kinds of items.

\section{Notes}

1. We have had some validation studies for our in-house tests. For example, we administered a Cambridge Exam (Otomo,1996) and a TOEFL-ITP (Hirai, et al., 2005) to the students, in order to examine reliabilities, correlations and so forth between our test and these proficiency tests and establish a comparative table that equates scores on our test with scores on the Cambridge Exam and TOEFL tests. 
2. Bigsteps is a DOS-based Rasch measurement program prior to Winsteps. It is now downloaded for free (see http://www.winsteps.com/bigsteps.htm).

3. Test usefulness should be considered, in developing and implementing a test and assessing its test takers. Bachman and Palmer (1996) propose a model of test usefulness which consists of six test qualities: reliability, validity, authenticity, interactiveness, impact, and practicality. All of them contribute in unique but interrelated ways to the overall usefulness of a given test. Therefore, test developers need to find an appropriate balance among these qualities.

\section{Acknowledgements}

I would like to thank all the members of the Foreign Language Center at the University of Tsukuba. Especially, I am indebted to E. Hamana for arranging the Access workshop in 2002, and the members of the Tsukuba proficiency test analysis section, H. Iwasaki, M. Shimada, M. Kaigo, and T. Takagi, for their valuable feedback regarding the usability of the item bank while they worked with the item bank.

\section{References}

ALTE Working Group (2001). Principles of good practice for ALTE examinations. pp.1-19, http://www.alte.org/quality_assurance/code/good_practice.pdf. Bachman, L. F., \& Palmer, A. S. (1996). Language Testing in Practice. Oxford University Press.

Beeston, S. (2000). The UCLES EFL item banking system. Research Notes - Issue 2, August 2000. http://www.cambridgeesol.org/rs_notes/0002/rs_notes2_4.cfm Henning, G. (1986). Item banking via DBASE II: the UCLA ESL Proficiency Examination experience. In Charles W. Stansfield (Ed.), Technology and Language Testing. pp. 69-78. Washington, D.C.: TESOL Publications.

Hirai, A. \& Shillaw, J. (2002). 3.5.2. Tsukuba Eigo Kentei Shaken ni yoru eigoryoku no genjyo [3.5.2. The English proficiency level of Tsukuba students based on Tsukuba English Proficiency Test]. In A. Kawasaki (Ed.) Tsukubadaigaku no Eigokyouiku [English education at the University of Tsukuba]. Studies in Language and Culture 59. (pp.154-160). Tsukuba: University of Tsukuba. Hirai, A. Mochizuki, A., Kubota, A., \& Iwasaki, S. (2005). Tsukuba Daigaku no Eigo 
Kentei Shiken to TOEFL [Tsukuba English Proficiency Test and TOEFL]. In E. Hamana (Ed.) TOEFL-ITP no Shikenteki Gisshi Data niyoru Gakunai Eigo Kentei Shiken no Kaizen [Assessing improvements of Tsukuba Proficiency Test based on the empirical data of TOEFL-ITP], pp.7-18. Tsukuba: University of Tsukuba, Foreign Language Center.

Linacre, J. M. \& Wright, B. D. (1997). A User's Guide to BIGSTEPS. Chicago, IN: MESA Press.

Machida, N. (2001). ACCESS 2002: Basic operation. Tokyo:Gijutsuhyoronsha

McNamara, T. F. (1996). Measuring Second Language Performance. London:

Longman.

Rudner, L. (1998). Item banking. Practical assessment, research \& evaluation, 6(4).

Retrieved July 10, 2005 from http://PAREonline.net/getvn.asp?v=6\& $=4$

Smith, R., Sussman, D., Blackburn, I., Colby, J., Horner, M., Reid, M., Turley, P. \&

Watson, H. (2003). Beginning Access 2002 VBA. Indianapolis, IN: Wiley Publishing, Inc. 